\title{
Echocardiographic features of patients with COVID-19 infection: a cross-sectional study
}

\author{
Hasan Ali Barman ${ }^{1,4}$ (1) Adem Atici $^{2} \cdot$ Esra Aktas Tekin $^{3} \cdot$ Omer Faruk Baycan ${ }^{2} \cdot$ Gokhan Alici $^{4}$. \\ Bengisu Keskin Meric ${ }^{1} \cdot$ Omer $\mathrm{Sit}^{4} \cdot$ Omer Genc $^{5} \cdot$ Fahri Er $^{5} \cdot$ Baris Gungor $^{6} \cdot$ Irfan Sahin $^{7} \cdot$ Namigar Turgut $^{3}$
}

Received: 16 August 2020 / Accepted: 29 September 2020 / Published online: 8 October 2020

(c) Springer Nature B.V. 2020

\begin{abstract}
COVID-19 patients with cardiac involvement have a high mortality rate. The aim of this study was to investigate the echocardiographic features in COVID-19 patients between severe and non-severe groups.For this single-center study, data from patients who were treated for COVID-19 between March 25, 2020 and April 15, 2020 were collected. Two-dimensional echocardiography (2DE) images were obtained for all patients. Patients were divided into two groups based on the severity of their COVID-19 infections. 2DE parameters indicating right ventricular (RV) and left ventricular (LV) functions were compared between the two groups.A total of 90 patients hospitalized for COVID-19 were included in this study. The mean age of the severe group $(n=44)$ was $63.3 \pm 15.7$ years, and $54 \%$ were male. The mean age of non-severe group $(n=46)$ was $49.7 \pm 21.4$ years, and $47 \%$ were male. In the severe group, $\mathrm{RV}$ and $\mathrm{LV}$ diameters were larger (RV, $36.6 \pm 5.9 \mathrm{~mm}$ vs. $33.1 \pm 4.8 \mathrm{~mm}, \mathrm{p}=0.003 ; \mathrm{LV} 47.3 \pm 5.8 \mathrm{~mm}$ vs. $44.9 \pm 3.8 \mathrm{~mm}, \mathrm{p}=0.023$ ), the LE ejection fraction (LVEF) and the RV fractional area change (RV-FAC) were lower (LVEF, $54.0 \pm 9.8 \%$ vs. $61.9 \pm 4.8 \%, \mathrm{p}<0.001$; RV-FAC, $41.4 \pm 4.1 \%$ vs. $45.5 \pm 4.5 \%, \mathrm{p}<0.001)$, and pericardial effusions were more frequent ( $23 \%$ vs. $0 \%$ ) compared to patients in the non-severe group. A multiple linear regression analysis determined that LVEF, right atrial diameter, high-sensitivity troponin I, d-dimer, and systolic pulmonary artery pressure, were independent predictors of RV dilatation. The results demonstrate that both right and left ventricular functions decreased due to COVID-19 infection in the severe group. 2DE is a valuable bedside tool and may yield valuable information about the clinical status of patients and their prognoses.
\end{abstract}

Keywords COVID-19 · Two-dimensional echocardiography · Cardiac injury

Hasan Ali Barman

drhasanali@hotmail.com

1 Institute of Cardiology, Department of Cardiology, Istanbul University, Cerrahpasa, Istanbul, Turkey

2 Faculty of Medicine, Department of Cardiology, Goztepe Training and Research Hospital, Istanbul Medeniyet University, Istanbul, Turkey

3 Department of Anesthesiology and Intensive Care, Okmeydani Training and Research Hospital, University of Health Sciences, Istanbul, Turkey

4 Department of Cardiology, Okmeydani Training and Research Hospital, University of Health Sciences, Istanbul, Turkey

5 Department of Cardiology, Agri State Hospital, Agri, Turkey

6 Department of Cardiology, Dr. Siyami Ersek Training and Research Hospital, University of Health Sciences, Istanbul, Turkey

7 Department of Cardiology, Bagcilar Training and Research Hospital, University of Health Sciences, Istanbul, Turkey

\section{Introduction}

COVID-19 (Coronavirus Disease 2019), caused by severe acute respiratory syndrome-coronavirus-2 (SARS-CoV-2), is a disease with high mortality due to multi-organ failure involving the respiratory, cardiac, renal, neurological, hepatic and intestinal systems [1,2]. COVID-19 infection is associated with many direct or indirect cardiovascular complications, including myocarditis, myocardial damage, arrhythmia, heart failure (HF), and venous thromboembolism $[3,4]$, and myocardial ischemia and necrosis is associated with impaired ventricular function that causes an increased mortality risk in patients [5].

Two-dimensional echocardiography (2DE) is an important, noninvasive test which helps to identify cardiac function and hemodynamic status. 2DE has gained importance in COVID-19 patients with multi-organ involvement, especially in cases involving hemodynamic 
instability. Due to risk of infection, routine 2DE is not recommended for each patient, and there are currently no comprehensive 2DE studies in COVID-19 patients. Here, we investigated $2 \mathrm{DE}$ variables and their possible correlations with disease severity in COVID-19 patients.

\section{Methods}

\section{Study population}

Between March 25, 2020 and April 15, 2020, 90 patients with positive SARS-CoV-2 real-time RT-PCR tests were included. 2DE images were obtained for all patients. According to the severity of their COVID-19 infections, the patients were divided into two groups: severe and nonsevere. Patients younger than 18 years of age, patients with a previous history of HF, valvular heart disease, coronary artery disease, atrial fibrillation, pulmonary hypertension, pulmonary embolism, chronic obstructive pulmonary disease, and renal failure were excluded from the study. This study was approved by the institutional review board, and the Republic of Turkey Ministry of Health.

\section{Data collection}

Detailed data about patient demographics, clinical characteristics, and laboratory findings were retrospectively collected from medical records. The images from chest computed tomography (CT) scans were retrieved from the picture archiving and communication system (PACS). Laboratory results upon admission included complete blood cell analysis, blood biochemistry, kidney function, electrolytes, C-reactive protein (CRP), high-sensitivity troponin I (hs-TnI), and d-dimer.

\section{Transthoracic two-dimensional echocardiography}

2DE studies were performed by a cardiologist using an X5 transducer (Philips Epiq7; Philips Healthcare, Inc., Andover, MA, USA) to evaluate parasternal and apical images (2D, M-mode, Doppler echocardiography), with the patient placed in the left lateral decubitus position. After the 2DE images were recorded, analyses was performed by two independent and experienced cardiologists, blinded to the clinical data of the patients, at the end of the day. Echocardiographic images were obtained using the four standard views (long-axis parasternal, short-axis parasternal, two-chamber apical, and fourchamber apical) using the techniques recommended by the American Society of Echocardiography (ASE) guidelines [6]. Using the parasternal long-axis view, the left ventricular end diastolic diameter (LVEDD), the left ventricular end systolic diameter (LVESD), and the interventricular septal (IVS) and posterior wall (PW) thicknesses (just distal to the mitral leaflets tips) were expressed in millimeters. Left ventricular systolic function was assessed using the left ventricular ejection fraction (LVEF) that was calculated from apical 4- and 2 -chamber views by manually tracing end-diastolic and end-systolic endocardial borders, using Simpson's biplane method [7]. The left atrial (LA) diameter was measured from the parasternal long-axis view at end-systole. The right ventricular (RV) diameter was measured from the apical 4-chamber view at the tricuspid annulus, $42-45 \mathrm{~mm}$ at the base indicating right ventricular dilatation [8]. The upper normal limit for the long axis dimension of the right atrium (RA) is $45 \mathrm{~mm}$. Tricuspid annulus plane systolic excursion (TAPSE) was done by the M-Mode, which is placed across the lateral tricuspid valve annulus using a 4-chamber view. Systolic pulmonary artery pressure (sPAP) was measured by tricuspid regurgitation (TR) peak velocity. 2DE RV fractional area change (RV-FAC) was calculated using a 4-chamber view by tracing the end-diastolic and end-systolic cavity, and calculating the percentage change in area. Tricuspid lateral annular systolic velocity ( $\left.\mathrm{S}^{\prime}\right)$ was assessed by pulse wave (PW) tissue Doppler imaging (TDI) from a 4-chamber view by placing the sample volume at the base of the free RV wall. After each examination, the device probes were cleaned with hydrogen peroxide disposable wipes. When an echocardiography device entered the COVID-19 service, it remained in that service and was not shared with other departments of the hospital.

\section{Definitions}

According to the World Health Organization (WHO) interim guidance, the diagnosis of COVID-19 is based on a real-time reverse-transcription polymerase chain reaction (RT-PCR) test. SARS-CoV-2 RNA was detected using the real-time RT-PCR method in the Public Health Microbiology Reference Laboratory of the Ministry of Health. The severe COVID-19 group was defined with any of the following: (1) respiratory distress (respiratory rate $\geq 30$ breaths per min); (2) oxygen saturation at rest $\leq 93 \%$; (3) ratio of the partial pressure of arterial oxygen $\left(\mathrm{PaO}_{2}\right)$ to the fractional concentration of oxygen inspired air $\left(\mathrm{FiO}_{2}\right)\left(\mathrm{PaO}_{2}: \mathrm{FiO}_{2}, \leq 300 \mathrm{mmHg}\right)$; or (4) a critical complication (respiratory failure and mechanical ventilation required, septic shock, and/or multiple organ dysfunction/failure and intensive care unit admission required) [9]. Acute cardiac injury was defined as hs-TnI 
Table 1 Demographic and clinical characteristics of patients severe and non-severe

\begin{tabular}{|c|c|c|c|}
\hline & Severe $(n=44)$ & Non-severe $(n=46)$ & $p$ \\
\hline Age (years) & $63.3 \pm 15.7$ & $49.7 \pm 21.4$ & 0.001 \\
\hline Male, n (\%) & $24(54 \%)$ & $22(47 \%)$ & 0.524 \\
\hline $\mathrm{BMI}\left(\mathrm{kg} / \mathrm{m}^{2}\right)$ & $30.1 \pm 5.6$ & $28.1 \pm 5.3$ & 0.224 \\
\hline Fever, n (\%) & $23(52 \%)$ & $22(48 \%)$ & 0.546 \\
\hline Cough, n (\%) & $29(66 \%)$ & $28(61 \%)$ & 0.614 \\
\hline Shortness of breath, $\mathrm{n}(\%)$ & $20(45 \%)$ & $7(15 \%)$ & $<0.001$ \\
\hline $\mathrm{HT}, \mathrm{n}(\%)$ & $23(52 \%)$ & $9(19 \%)$ & 0.001 \\
\hline $\mathrm{DM}, \mathrm{n}(\%)$ & $10(22 \%)$ & $4(8 \%)$ & 0.066 \\
\hline HLD, n (\%) & $7(15 \%)$ & $3(6 \%)$ & 0.196 \\
\hline Smoking, n (\%) & $26(\% 59)$ & $25(\% 55)$ & 0.754 \\
\hline $\mathrm{HR}$, beats/min & $78.1 \pm 13.8$ & $72.9 \pm 12.6$ & 0.072 \\
\hline $\mathrm{SBP}(\mathrm{mmHg})$ & $104.4 \pm 10.9$ & $111.3 \pm 11.0$ & 0.093 \\
\hline $\mathrm{DBP}(\mathrm{mmHg})$ & $70.9 \pm 6.4$ & $69.7 \pm 8.4$ & 0.464 \\
\hline \multicolumn{4}{|c|}{ Laboratory findings on admission } \\
\hline Hemoglobin (g/Ll) & $11.3 \pm 2.3$ & $13.5 \pm 1.8$ & $<0.001$ \\
\hline $\mathrm{WBC}\left(10^{3} / \mu \mathrm{L}\right)$ & $7.0(5.2-12.0)$ & $5.4(3.6-7.3)$ & 0.005 \\
\hline Creatinine (mg/dL) & $0.8(0.6-1.2)$ & $0.8(0.6-0.9)$ & 0.563 \\
\hline Sodium (mmol/L) & $139.3 \pm 6.4$ & $137.3 \pm 2.7$ & 0.070 \\
\hline Potassium (mmol/L) & $4.0 \pm 0.6$ & $4.2 \pm 0.4$ & 0.239 \\
\hline Glucose (mg/dL) & $147.9 \pm 60.8$ & $117.8 \pm 40.2$ & 0.008 \\
\hline $\mathrm{CRP}(\mathrm{mg} / \mathrm{dL})$ & $102(40-188)$ & $20(10-81)$ & $<0.001$ \\
\hline Hs-TnI (pg/mL) & $20(5-86)$ & $9(3-16)$ & 0.004 \\
\hline D-dimer (ng/mL) & $1170(330-2840)$ & $255(27-510)$ & $<0.001$ \\
\hline CK-MB (ng/mL) & $2.2(1.1-3.8)$ & $1.3(0.9-2.3)$ & 0.083 \\
\hline $\mathrm{O}_{2}$ saturation, $\% *$ & $87.5 \pm 3.6$ & $95.5 \pm 1.9$ & $<0.001$ \\
\hline \multicolumn{4}{|l|}{ Clinical parameters } \\
\hline ICU, n (\%) & $29(65 \%)$ & - & - \\
\hline $\mathrm{MV}, \mathrm{n}(\%)$ & $24(54 \%)$ & - & - \\
\hline Hospital stay** (days) & $12.2 \pm 4.3$ & $8.0 \pm 4.3$ & $<0.001$ \\
\hline Pneumonia on CT, n (\%) & $43(97 \%)$ & $37(80 \%)$ & 0.009 \\
\hline
\end{tabular}

$B M I$ Body mass index, $H T$ hypertension, $D M$ diabetes mellitus, $H L D$ hyperlipidemia, $H R$ heart rate, $S B P$ systolic blood pressure, $D B P$ diastolic blood pressure, $W B C$ white blood cell, $C R P$ C-reactive protein, $h s-T n I$ high sensitive troponin I, $C K$ creatinine kinase, ICU intensive care unit, $M V$ mechanical ventilation, CT computed tomography

*Reported for patients without MV

** Days from admission to the day of echocardiographic examination serum levels above the 99th percentile upper reference limit [10].

\section{Statistical analyses}

All statistical tests were conducted using the Statistical Package for the Social Sciences 21.0 for Windows (SPSS Inc., Chicago, IL, USA). The Kolmogorov-Smirnov test was used to analyze normality of the data. Continuous data are expressed as mean $\pm \mathrm{SD}$, and categorical data are expressed as percentages. The Chi-square test was used to assess differences in categorical variables between groups. Student's t-test or the Mann-Whitney U test was used to compare unpaired samples as needed. The relationships among parameters were assessed using Pearson's or Spearman's correlation analysis according to the normality of the data. 15 patients were randomly selected to assess intra- and inter-observer variability for LVEF, RV-FAC, and the RV-diameter, expressed as intraclass correlation coefficients (ICCs). Multiple linear regression analyses using the stepwise method were performed to assess the independent variables affecting the dependent variable (RV diameter). All independent variables in the multiple linear regression were tested for multicollinearity. If the variance inflation factor (VIF) exceeded 3.0, the variable was considered to be collinear. All reported confidence interval (CI) values were calculated at the $95 \%$ level. Significance was assumed using a two-sided $\mathrm{p}$ value $<0.05$. 
Table 2 Comparison of 2-D transthoracic echocardiographic parameters in the study population

\begin{tabular}{|c|c|c|c|c|c|}
\hline & Non-severe $(n=46)$ & Severe $(n=44)$ & $\begin{array}{l}\text { Severe exc. } \\
\text { MV }(n=20)\end{array}$ & $\mathrm{p}^{1}$ & $\mathrm{p}^{2}$ \\
\hline \multicolumn{6}{|l|}{ Left heart findings } \\
\hline $\operatorname{LVEF}(\%)$ & $61.9 \pm 4.8$ & $54.0 \pm 9.8$ & $58.7 \pm 7.4$ & $<0.001$ & 0.041 \\
\hline $\operatorname{LVEDD}(\mathrm{mm})$ & $44.9 \pm 3.8$ & $47.3 \pm 5.8$ & $48.3 \pm 5.2$ & 0.023 & 0.005 \\
\hline LVESD (mm) & $28.8 \pm 4.1$ & $33.1 \pm 6.7$ & $32.8 \pm 6.5$ & 0.001 & 0.004 \\
\hline LV mass, $\mathrm{g} / \mathrm{m}^{2}$ & $92.9 \pm 4.5$ & $95.0 \pm 6.2$ & $94.2 \pm 5.4$ & 0.076 & 0.333 \\
\hline $\mathrm{LA}(\mathrm{mm})$ & $34.6 \pm 5.5$ & $39.4 \pm 5.5$ & $38.8 \pm 5.2$ & $<0.001$ & 0.005 \\
\hline $\mathrm{E}(\mathrm{cm} / \mathrm{s})$ & $90.6 \pm 25.4$ & $67.8 \pm 13.6$ & $66.5 \pm 13.6$ & $<0.001$ & $<0.001$ \\
\hline $\mathrm{A}(\mathrm{cm} / \mathrm{s})$ & $69.7 \pm 16.2$ & $74.8 \pm 14.6$ & $69.5 \pm 13.6$ & 0.130 & 0.965 \\
\hline E/A ratio & $1.2 \pm 0.5$ & $0.9 \pm 0.3$ & $1.0 \pm 0.4$ & $<0.001$ & 0.041 \\
\hline \multicolumn{6}{|l|}{ Right heart findings } \\
\hline $\mathrm{RV}(\mathrm{mm})$ & $33.1 \pm 4.8$ & $36.6 \pm 5.9$ & $36.7 \pm 5.2$ & 0.003 & 0.010 \\
\hline $\mathrm{RV} \geq 42 \mathrm{~mm}, \mathrm{n}(\%)$ & $2(4 \%)$ & $11(25 \%)$ & $3(15 \%)$ & 0.007 & 0.159 \\
\hline $\mathrm{RA}(\mathrm{mm})$ & $36.8 \pm 6.6$ & $39.9 \pm 7.3$ & $41.7 \pm 5.9$ & 0.023 & 0.007 \\
\hline TAPSE (mm) & $21.4 \pm 3.6$ & $20.1 \pm 4.3$ & $20.3 \pm 4.3$ & 0.126 & 0.288 \\
\hline TAPSE $\leq 16$ mm, n (\%) & $4(8 \%)$ & $11(25 \%)$ & $3(15 \%)$ & 0.016 & 0.425 \\
\hline RV-FAC, $\%$ & $45.5 \pm 4.5$ & $41.4 \pm 4.1$ & $41.5 \pm 3.4$ & $<0.001$ & 0.001 \\
\hline TDI S', $\mathrm{cm} / \mathrm{s}$ & $13.8 \pm 3.0$ & $13.1 \pm 3.0$ & $13.3 \pm 3.6$ & 0.324 & 0.585 \\
\hline $\mathrm{PA}, \mathrm{mm}$ & $21.3 \pm 3.0$ & $21.3 \pm 3.0$ & $21.4 \pm 4.9$ & 0.413 & 0.976 \\
\hline sPAP, mmHg & $28.5 \pm 7.3$ & $35.5 \pm 8.6$ & $32.0 \pm 9.3$ & 0.039 & 0.117 \\
\hline $\mathrm{sPAP} \geq 35 \mathrm{mmHg}, \mathrm{n}(\%)$ & $6(14 \%)$ & $17(38 \%)$ & $8(40 \%)$ & 0.013 & 0.027 \\
\hline IVC (mm) & $12.5 \pm 2.6$ & $16.8 \pm 5.0$ & $12.8 \pm 3.5$ & $<0.001$ & 0.689 \\
\hline Pericardial effusion, $\mathrm{n}(\%)$ & $0(0 \%)$ & $10(23 \%)$ & $1(5 \%)$ & - & - \\
\hline
\end{tabular}

$M V$ mechanical ventilation, $L V E F$ left ventricular ejection fraction, $L V E D D$ left ventricular end diastolic diameter, $L V E S V$ left ventricular end systolic diameter, $L A$ left atrial, $M R$ mitral regurgitation, $R V$ right ventricular, $R A$ right atrial, TAPSE tricuspid annular plane systolic excursion, $R V-F A C$ right ventricular fractional area change, $T D I S$ ' tissue Doppler imaging systolic wave $S$ ' velocity, $P A$ pulmonary artery, $T R$ tricuspid regurgitation, $S P A P$ systolic pulmonary artery pressure, $I V C$ inferior vena cava

$\mathrm{p}^{1}$ comparison between non-severe and severe groups

$\mathrm{p}^{2}$ comparison between non-severe and severe groups excluding patients with mechanical ventilation

\section{Results}

The demographic, clinical and laboratory parameters of the 90 patients included in the study are shown in Table 1 . The severe group included 44 patients who were older compared to the 46 non-severe patients. There were no statistically significant differences between the groups in terms of gender, body mass index (BMI), diabetes mellitus (DM), hyperlipidemia, and smoking, while hypertension (HT) was more frequent in the severe group. There were no statistical differences between the two groups in terms of heart rate, systolic and diastolic blood pressure, creatinine, sodium, potassium, and creatine-kinase MB (CK-MB) levels. However, hemoglobin, white blood cell (WBC), glucose, CRP, hs-TnI, and d-dimer levels were significantly different between the groups. In the severe group, typical pneumonia was more frequently encountered on CT images $(97 \%$ vs. $80 \%$ ) and patients in this group had lower $\mathrm{O}_{2}$ saturations.

The comparison of 2DE examination findings is shown in Table 2. LVEDD, LVESD, and LA diameters were higher, and LVEF, E wave, E/A ratios, and RV-FAC were lower in the severe patient group. RV, RA, inferior vena cava (IVC) diameters were increased, and sPAP was higher in the severe group, but TAPSE values were similar between the groups. Figures 1 and 2 show sample echocardiographic images from the severe and the non-severe group. Pericardial effusion were also more frequent in the severe patient group (23\% vs. $0 \%)$.

When patients requiring mechanical ventilation (MV) were removed from the analysis, patients in the severe group still had increased LVEDD, LVESD, RV, RA, and LA diameters, and decreased LVEF, E wave, E/A ratios and RV-FAC compared to the non-severe group (Table 2). However, none of the echocardiographic indices differed significantly according to the type of ventilation within the severe group ( $p>0.05$ for all comparisons).

In a subgroup analysis, 29 patients with concomitant myocardial injuries were compared to 61 patients without myocardial injuries. Patients with myocardial injuries were older $(66.7 \pm 15.6$ years vs. $51.5 \pm 20.1$ years $)$, had higher 
Table 3 Transthoracic echocardiography parameters of patients with and without cardiac injury

\begin{tabular}{|c|c|c|c|}
\hline & $\begin{array}{l}\text { Cardiac } \\
\text { injury }(+) \\
(\mathrm{n}=29)\end{array}$ & $\begin{array}{l}\text { Cardiac } \\
\text { injury }(-) \\
(\mathrm{n}=61)\end{array}$ & $\mathrm{p}$ \\
\hline \multicolumn{4}{|l|}{ Left heart findings } \\
\hline $\operatorname{LVEF}(\%)$ & $52.3 \pm 10.8$ & $60.8 \pm 5.7$ & $<0.001$ \\
\hline LVEDD (mm) & $46.0 \pm 6.3$ & $46.1 \pm 4.3$ & 0.899 \\
\hline LVESD (mm) & $32.7 \pm 7.4$ & $30.1 \pm 4.9$ & 0.054 \\
\hline IVS (mm) & $11.2 \pm 1.6$ & $10.3 \pm 2.4$ & 0.084 \\
\hline PW (mm) & $10.6 \pm 1.3$ & $9.5 \pm 2.2$ & 0.020 \\
\hline LA (mm) & $37.7 \pm 5.0$ & $36.6 \pm 6.4$ & 0.417 \\
\hline $\mathrm{E}(\mathrm{cm} / \mathrm{s})$ & $69.5 \pm 16.6$ & $84.3 \pm 24.7$ & 0.002 \\
\hline $\mathrm{A}(\mathrm{cm} / \mathrm{s})$ & $77.2 \pm 16.1$ & $69.9 \pm 14.9$ & 0.043 \\
\hline E/A ratio & $0.9 \pm 0.2$ & $1.2 \pm 0.5$ & 0.003 \\
\hline \multicolumn{4}{|l|}{ Right heart findings } \\
\hline $\mathrm{RV}(\mathrm{mm})$ & $39.5 \pm 6.0$ & $34.0 \pm 5.3$ & 0.031 \\
\hline $\mathrm{RV} \geq 42 \mathrm{~mm}, \mathrm{n}(\%)$ & $8(27 \%)$ & $5(8 \%)$ & 0.014 \\
\hline $\mathrm{RA}(\mathrm{mm})$ & $37.2 \pm 6.4$ & $38.9 \pm 7.4$ & 0.300 \\
\hline TAPSE (mm) & $20.2 \pm 4.5$ & $21.1 \pm 3.6$ & 0.317 \\
\hline TAPSE $\leq 16 \mathrm{~mm}, \mathrm{n}(\%)$ & $9(31 \%)$ & $6(10 \%)$ & 0.014 \\
\hline RV-FAC, \% & $40.9 \pm 4.5$ & $44.8 \pm 4.9$ & 0.002 \\
\hline TDI S', cm/s & $13.4 \pm 2.9$ & $13.5 \pm 3.1$ & 0.926 \\
\hline $\mathrm{PA}, \mathrm{mm}$ & $21.5 \pm 3.9$ & $20.8 \pm 3.5$ & 0.383 \\
\hline sPAP, mmHg & $32.1 \pm 9.0$ & $29.1 \pm 7.5$ & 0.109 \\
\hline $\mathrm{sPAP} \geq 35 \mathrm{mmHg}, \mathrm{n}(\%)$ & $11(37 \%)$ & $12(19 \%)$ & 0.063 \\
\hline IVC (mm) & $19.0 \pm 4.6$ & $13.2 \pm 3.4$ & $<0.001$ \\
\hline Pericardial effusion, $\mathrm{n}(\%)$ & $8(27 \%)$ & $2(3 \%)$ & 0.001 \\
\hline
\end{tabular}

$L V E F$ left ventricular ejection fraction, $L V E D D$ left ventricular end diastolic diameter, $L V E S V$ left ventricular end systolic diameter, IVS interventricular septum, $P W$ posterior wall, $L A$ left atrial, $M R$ mitral regurgitation, $R V$ right ventricular, $R A$ right atrial, TAPSE tricuspid annular plane systolic excursion, $R V-F A C$ right ventricular fractional area change, TDI $S$ ' tissue Doppler imaging systolic wave $S$ ' velocity, $P A$ pulmonary artery, $T R$ tricuspid regurgitation, $s P A P$ systolic pulmonary artery pressure, $I V C$ inferior vena cava

hs-TnI and d-dimer levels, and required more frequent ICU admissions (69\% vs. 18\%). When echocardiography parameters were compared, LVEDD, LVESD, RV diameters $(39.5 \pm 6.0 \mathrm{~mm}$ vs. $34.0 \pm 5.3 \mathrm{~mm})$ were significantly higher and LVEF $(52.3 \pm 10.8 \%$ vs. $60.8 \pm 5.7 \%)$ and RV-FAC were significantly lower in patients with myocardial injury. However, RA diameters and TAPSE values were comparable between the two groups. The frequency of pericardial effusion was significantly higher in the subgroup group with developed myocardial injury $(27 \%$ vs. $3 \% ; p=0.001)$. In none of the patients was pericardial tamponade diagnosed (Table 3).

The relationships between RV, LVEF and RV-FAC and hs-TnI, d-dimer and CRP levels were evaluated by univariate correlation analysis (Table 4). A significant positive correlation was found between RV diameter,
Table 4 Correlation of echocardiographic findings with prognostic laboratory parameters

\begin{tabular}{lrrr}
\hline Spearman & \multicolumn{1}{c}{ hs-TnI } & D-dimer & \multicolumn{1}{c}{ CRP } \\
\hline$R V$ diamater & & & \\
Correlation coefficient & 0.646 & 0.612 & 0.168 \\
$p$ & $<0.001$ & $<0.001$ & 0.119 \\
$L V E F$ & & & \\
Correlation coefficient & -0.388 & -0.464 & -0.310 \\
$p$ & $<0.001$ & $<0.001$ & 0.003 \\
$R V$-FAC & & & \\
Correlation Coefficient & -0.656 & -0.624 & -0.101 \\
$p$ & $<0.001$ & $<0.001$ & 0.353 \\
\hline
\end{tabular}

$R V$ right ventricular, $L V E F$ left ventricular ejection fraction, $R V-F A C$ right ventricular fractional area change, $h s-T n I$ high-sensitive troponin I, CRP C-reactive protein

hs-TnI, and d-dimer levels (Fig. 3) and LVEF was negatively correlated with hs-TnI, d-dimer, and CRP levels. Similarly, a statistically significant negative correlation was found between RV-FAC, hs-TnI, and d-dimer levels, but not with CRP levels.

The independent predictors of RV diameter were analyzed using a linear regression analysis. The parameters that were statistically significant, and the parameters likely to affect $\mathrm{RV}$ diameters, were included in the model. In this context, the relationships between age, HT, hemoglobin, creatinine, glucose, CRP, hs-TnI, d-dimer, LVEF, RA, sPAP, TAPSE, and RV diameter were evaluated. LVEF, RA, hs-TnI, d-dimer, and sPAP were defined as independently correlated with RV diameters (Table 5).

\section{Reproducibility}

Intra- and inter-observer reliability for LVEF, RV-FAC, and $\mathrm{RV}$-diameter values were assessed in 15 randomly selected subjects and expressed as ICCs. ICCs for intra- and interobserver reliability for LVEF were 0.90 (95\% CI 0.85-0.95) and 0.87 (95\% CI 0.81-0.94), respectively; for RV-FAC they were 0.91 (95\% CI 0.86-0.95) and 0.88 (95\% CI 0.82-0.95), respectively; and for RV-diameter they were 0.91 (95\% CI $0.85-0.96$ ) and 0.88 (95\% CI 0.83-0.93), respectively.

\section{Discussion}

The principal findings of our study were: (1) In COVID19 patients classified as severe, RV, LV, RA, and IVC diameters were higher, LVEF and RV-FAC were lower, and pericardial effusions were more frequent compared to non-severe patients. (2) A linear regression analysis 
Table 5 Factors related to RV diameter of COVID-19 patients in stepwise multiple linear regression analysis

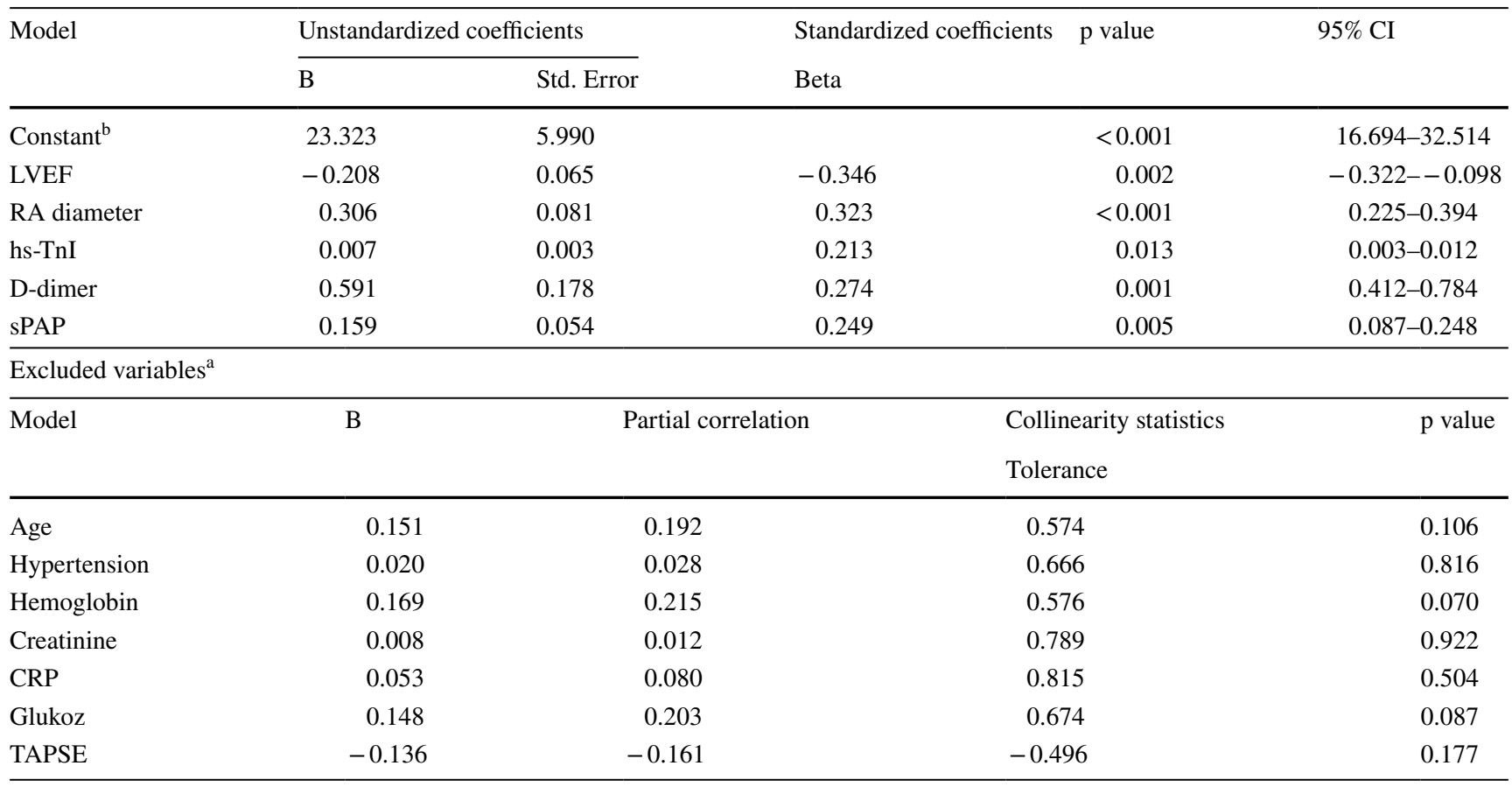

$R V$ right ventricular, $L V E F$ left ventricular ejection fraction, $R A$ right atrial, $h s$-TnI high-sensitive troponin I, $s P A P$ systolic pulmonary artery pressure, CRP C-reactive protein, TAPSE tricuspid annular plane systolic excursion

${ }^{a}$ Dependent variable: RV diameter

${ }^{\mathrm{b}}$ Correlates in the Model: (Constant), LVEF, RA diameter, hs-TnI, D-dimer, sPAP

determined that LVEF, RA diameter, hs-TnI, d-dimer, and sPAP were independent predictors of RV dilatation. (3) A correlation analysis showed significant correlations between echocardiographic parameters (RV diameter, LVEF, and RV-FAC) and laboratory parameters (elevated hs-TnI and d-dimer levels).

It is well-established that COVID-19 causes in multiorgan involvement, and the presence of cardiac dysfunction is associated with worse outcomes [11]. 2DE is very useful in this group of patients but is not routinely used due to the risk of transmitting the disease [12]. It allows noninvasive evaluation of cardiac function and hemodynamic status of the patient providing data on severity of disease [11]. In these patients, 2DE can also be used to exclude obstructive problems (e.g., cardiac tamponade and pulmonary embolism) and hypovolemic shock (decreased cardiac output and collapsed IVC) [13-15]. In recent years, studies have also revealed the importance of bedside 2DE in sepsis patients requiring MV $[16,17]$. In these studies, it was observed that approximately $30-40 \%$ of sepsis patients developed a decrease in LVEF and diastolic dysfunction. In sepsis patients, hyperdynamic heart functions due to systemic inflammatory responses, increased cardiac output (CO), and LVEF are observed in the early period. In the late period, extensive myocardial inhibition occurs due to severe hypoxia and inflammation [5].
Myocardial injury has been shown to be associated with worse prognoses in patients with COVID-19 [18, 19]. In these patients, myocardial involvement, segmental contraction defects or global hypokinesia may be observed in the left heart due to hypoxic damage, respiratory distress, and inflammation, and a recent report [20] has shown that even though LVEF was not decreased, a decrease in RV longitudinal strain (less than -23\%) may have a prognostic role in COVID-19 patients. In addition, Szekely et al. have demonstrated that patients with myocardial injury and worse clinical condition did not have any significant difference in LV systolic function, but had worse RV function compared to patients with normal troponin levels or mild clinical conditions [21]. Although our study also shows a decrease in RV-FAC, it diverges from the above study by showing a decrease in LVEF and enlargement of the LV. Hani et al. performed echocardiography on patients within five days of hospital admission, and showed normal or hyperdynamic LV systolic function with dilated and impaired RV function [22]. In contrast, we have shown that, both in the severe group and in patients with myocardial injury, LVEF and RV-FAC were decreased, the LV and RV were enlarged, and LV diastolic dysfunction was more frequent. Twenty-four out of the 44 severe patients were ventilated mechanically. We found that $\mathrm{RV}$ function (RV diameter and RV-FAC) and LVEF were still worse in the severe group of patients compared to the 


\section{A}

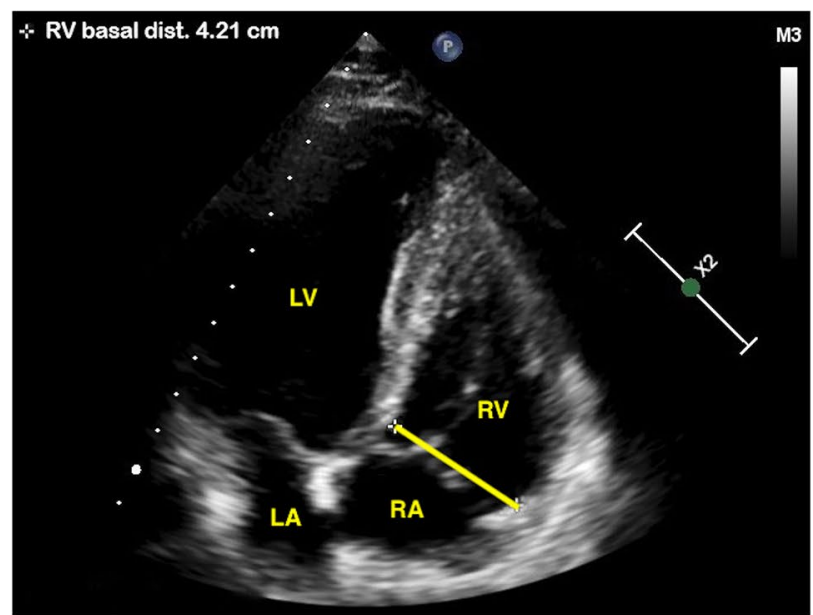

C

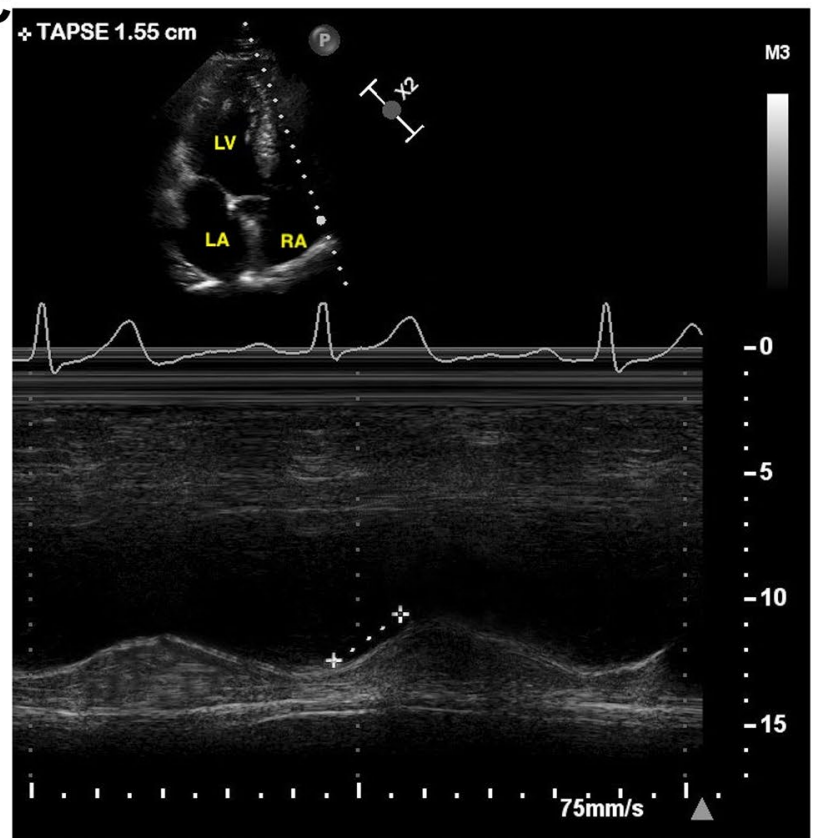

Fig. 1 a-d Two-dimensional transthoracic echocardiography of a patient with right ventricular dysfunction with severe COVID-19. a Enlarged right ventricle (RV). b Increased systolic pulmonary artery

non-severe group, but the echocardiographic indices were similar within the severe group regardless of the mode of ventilation.

In patients with COVID-19, alveolar damage, pulmonary capillary damage, and an increase in pulmonary vascular resistance all occur due to inflammation, hypoxia, and hypercapnia in the lungs. This causes an increase in RV afterload. Improper MV settings may also facilitate increases in pulmonary arterial pressure and RV afterload
B
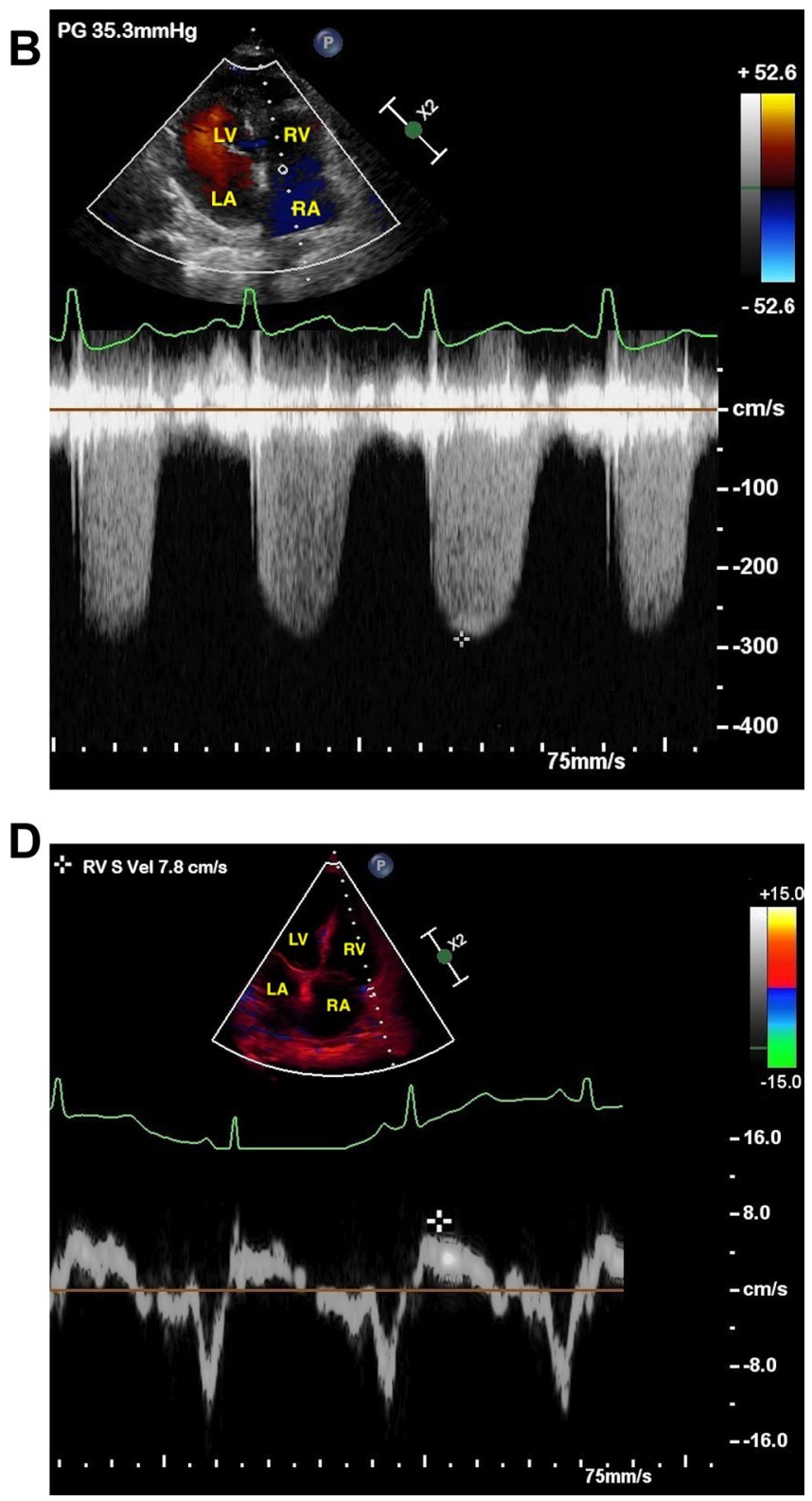

pressure (sPAP). c Reduced tricuspid annulus plane systolic excursion (TAPSE). d Decreased TDI-derived tricuspid lateral annular systolic velocity $\left(S^{\prime}\right)$

[5]. The right side of the heart's major function is circulation/oxygenation which is directly correlated with the clinical status of the COVID-19 patients. Development of $\mathrm{RV}$ dysfunction is part of a vicious cycle that causes deterioration in acute respiratory distress syndrome (ARDS) patients [23, 24]. The degree of RV dysfunction has been shown to be an important predictor of mortality in different patient groups. A significant portion of severe COVID-19 patients also needed MV, and such ventilation is known 
A

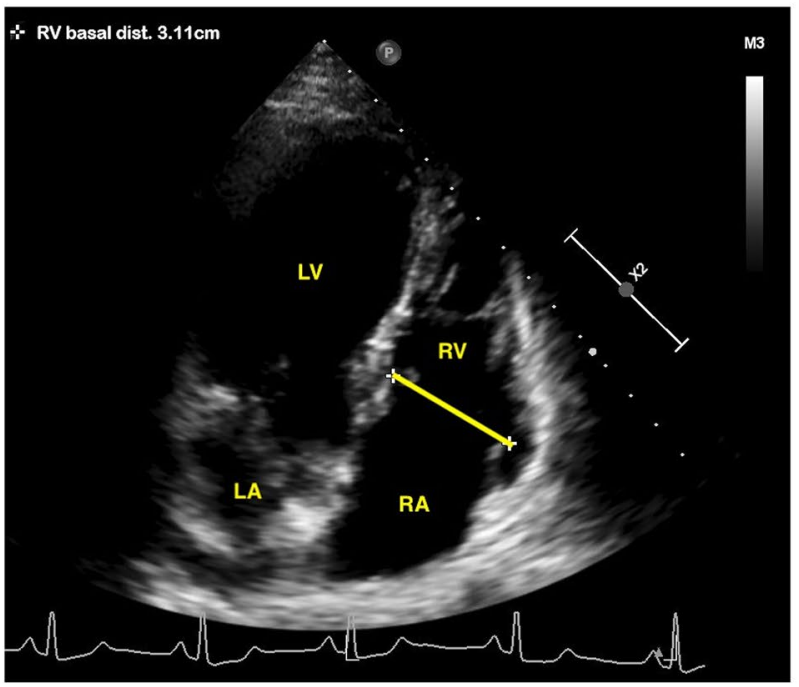

C

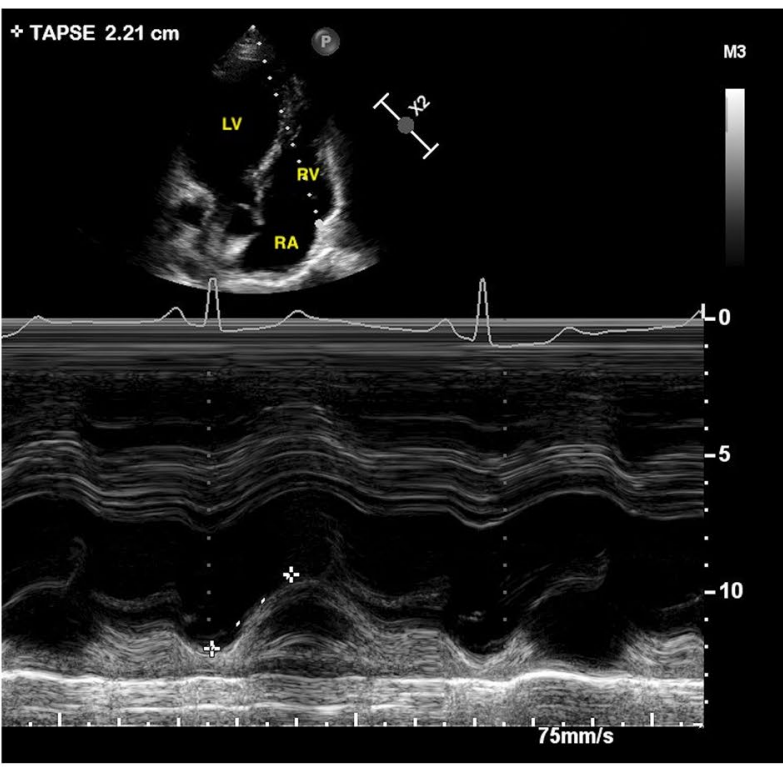

Fig. 2 a-d Two-dimensional transthoracic echocardiography of a patient with normal right ventricular function with non-severe COVID-19. a Right ventricle (RV). b Systolic pulmonary artery

to have hemodynamic effects on ventricular performance [25]. MV further increases pulmonary artery pressure and exacerbates right-heart dysfunction, especially if lungprotective ventilation is not performed properly. Postponing MV for as long as possible in each patient should be the standard in order to prevent the adverse effects of MV on ventricular function.

In COVID-19 patients, using 2DE is recommended to evaluate heart failure, arrhythmia, ECG changes, and newly
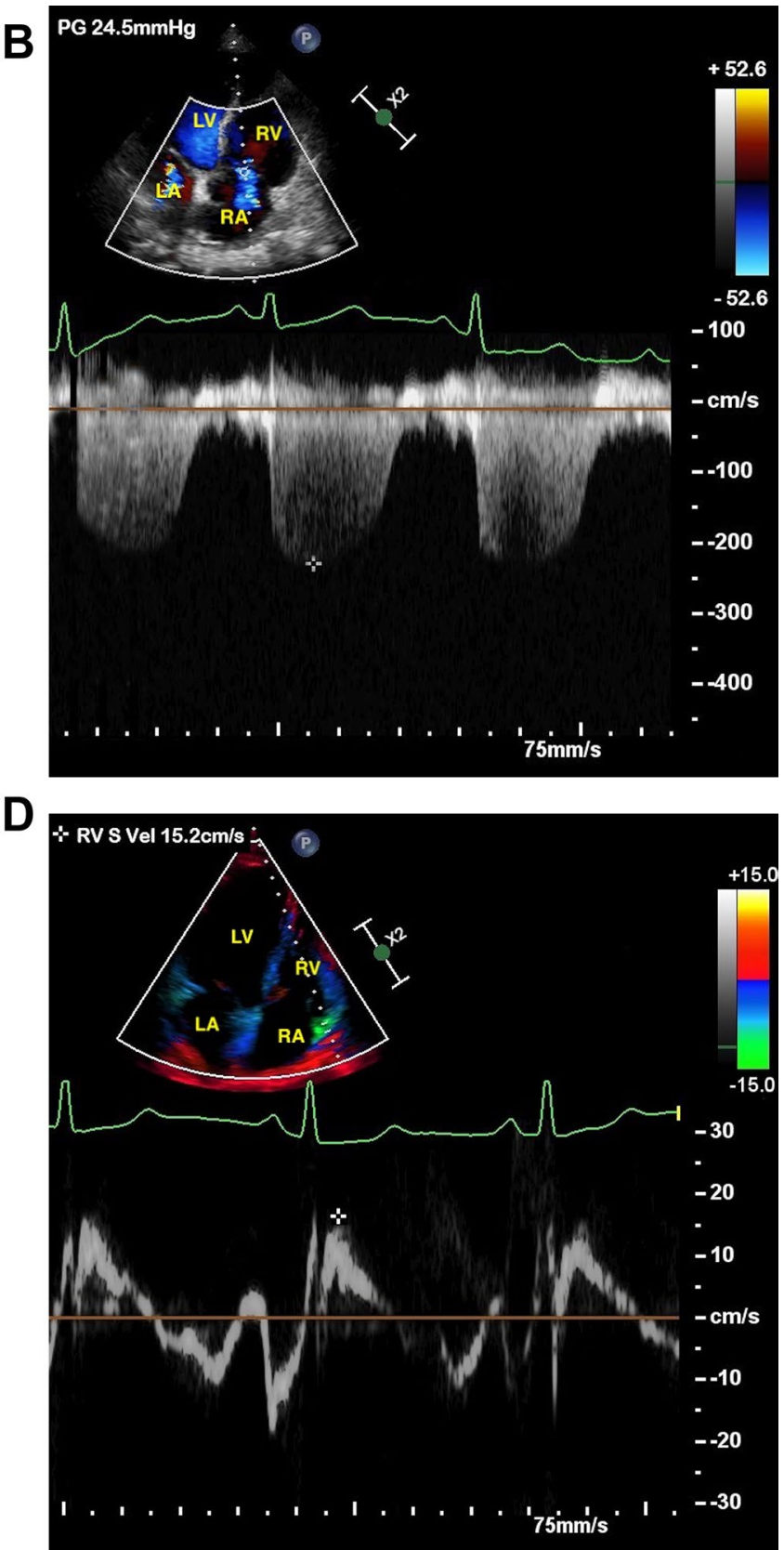

pressure (sPAP). c Tricuspid annulus plane systolic excursion (TAPSE). d TDI-derived tricuspid lateral annular systolic velocity $\left(\mathrm{S}^{\prime}\right)$

diagnosed cardiomegaly via chest X-ray or chest-CT [5]. According to ASE recommendations for COVID-19 patients [26], 2DE should be performed only if the test will make a significant contribution to the clinical course, and should not be repeated if there are no significant changes in patient progress. Personal protective equipment should be used, and contact time should be minimized. The decision to perform 2DE should be made by a combined evaluation of the patient's clinical status and all other tests. Non-urgent 

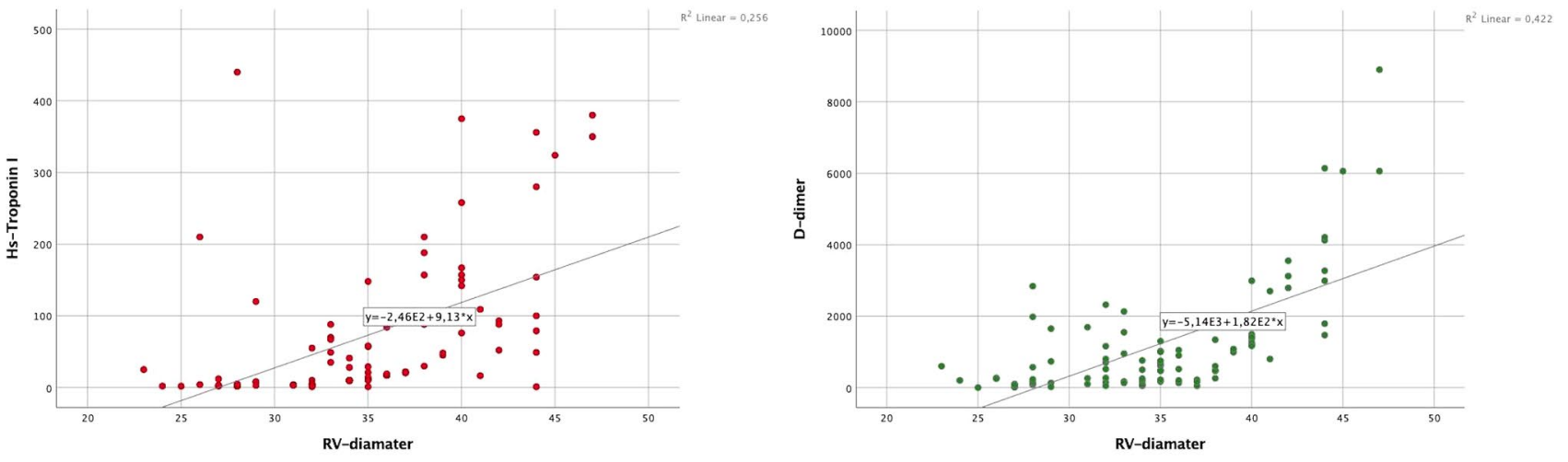

Fig. 3 Spearman Correlation analysis of RV diameter, hs-troponin I and d-dimer levels

echocardiography should be postponed for suspected or diagnosed COVID-19-positive patients.

Our study was limited in several ways. First, the principal limitation in the design of the study was the lack of a control group. Second, the data were from a single center, and the sample size was relatively small. Third, the lack measurements for cardiac output, cardiac index, and longitudinal strains for the LV and the RV. Fourth, there was no 2DE patient data prior to their COVID-19 infections, and there were no follow-up data related to echocardiography parameters. Fifth, the echocardiography images were recorded by one person due to the risk of infection, but evaluated by two physicians.

Here, we found that patients with COVID-19 may have biventricular systolic dysfunction in severe forms of the disease, especially in patients with cardiac injury. Echocardiographic parameters such as LVEF, RV diameter, and RV-FAC were correlated with laboratory parameters for bad prognoses such as elevated hs-TnI, CRP, and d-dimer. A 2DE examination can detect early signs of ventricular dysfunction, and this ability may help to guide therapy in this group of patients. 2DE may also be useful for detecting regional or global contraction defects of the LV in selected patients and for demonstrating acute overload of the RV in the presence of ARDS, especially in the presence of hemodynamic instability.

Funding The authors received no financial support for the research, authorship, and/or publication of this article.

\section{Compliance with ethical standards}

Conflict of interest The authors declare that they have no conflict of interest.

\section{References}

1 Feng Y, Ling Y, Bai T, Xie Y, Huang J, Li J et al (2020) COVID19 with different severity: a multi-center study of clinical features. Am J Respir Crit Care Med. 201:1380-1381

2. Organization WH (2020) Naming the coronavirus disease (COVID-19) and the virus that causes it. World Health Organization. https://www. who.int/emergencies/diseases/novel -coronavirus-2019/technical-guidance/naming-the-coronaviru s-disease- (covid-2019)-and-the-virus-that-causes-it

3 Akhmerov A, Marbán E (2020) COVID-19 and the heart. Circ Res 126 (10):1443-1455

4 Bansal M (2020) Cardiovascular disease and COVID-19. Diab Metab Syndr. https://doi.org/10.1016/j.dsx.2020.03.013

5 Peng Q-Y, Wang X-T, Zhang L-N (2020) Using echocardiography to guide the treatment of novel coronavirus pneumonia. Crit Care. https://doi.org/10.1186/s13054-020-02856-z

6 Lang RM, Badano LP, Mor-Avi V, Afilalo J, Armstrong A, Ernande L et al (2015) Recommendations for cardiac chamber quantification by echocardiography in adults: an update from the American Society of Echocardiography and the European Association of Cardiovascular Imaging. Eur Heart J Cardiovasc Imaging 16 (3):233-271

7. Schiller NB, Acquatella H, Ports TA, Drew D, Goerke J, Ringertz H et al (1979) Left ventricular volume from paired biplane two-dimensional echocardiography. Circulation 60 (3):547-555

8. Lai WW, Gauvreau K, Rivera ES, Saleeb S, Powell AJ, Geva T (2008) Accuracy of guideline recommendations for two-dimensional quantification of the right ventricle by echocardiography. Int J Cardiovasc Imaging 24 (7):691-698

9 Holshue ML, DeBolt C, Lindquist S, Lofy KH, Wiesman J, Bruce $\mathrm{H}$ et al (2020) First case of 2019 novel coronavirus in the United States. N Engl J Med. 382:929-936

10 Thygesen K, Alpert JS, Jaffe AS, Chaitman BR, Bax JJ, Morrow DA et al (2018) Fourth universal definition of myocardial infarction. Eur Heart J 40 (3):237-269

11 Lippi G, Lavie CJ, Sanchis-Gomar F (2020) Cardiac troponin I in patients with coronavirus disease 2019 (COVID19): evidence from a meta-analysis. Prog Cardiovasc Dis. 63:390-391

12 Picard MH, Weiner RB (2020) Echocardiography in the time of COVID-19. J Am Soc Echocardiogr. https://doi.org/10.1016/j. echo.2020.04.011 
13 Nagre AS (2019) Focus-assessed transthoracic echocardiography: implications in perioperative and intensive care. Ann Card Anaesth 22 (3):302

14 Perera P, Mailhot T, Riley D, Mandavia D (2010) The RUSH exam: rapid ultrasound in shock in the evaluation of the critically 11l. Emerg Med Clin 28 (1):29-56

15 Zhang L-N, Zhang H-M, Cao Y-G, Yin W-H, He W, Zhu R et al (2017) Ten basic principles about critical ultrasonography: critical care practitioners need to know. Chin Med J 130 (13): 1610

16 Bouhemad B, Nicolas-Robin A, Arbelot C, Arthaud M, Féger F, Rouby J-J (2009) Acute left ventricular dilatation and shockinduced myocardial dysfunction. Crit Care Med 37 (2):441-447

17 Vieillard-Baron A, Caille V, Charron C, Belliard G, Page B, Jardin F (2008) Actual incidence of global left ventricular hypokinesia in adult septic shock. Crit Care Med 36 (6):1701-1706

18 Barman HA, Atici A, Sahin I, Alici G, Tekin EA, Baycan ÖF et al (2020) Prognostic significance of cardiac injury in COVID19 patients with and without coronary artery disease. Coronary Artery Dis. https://doi.org/10.1097/MCA.0000000000000914

19. Frattini S, Maccagni G, Italia L, Metra M, Danzi GB (2020) Coronavirus disease 2019 and cardiovascular implications. J Cardiovasc Med 21 (10):725-732

20 Li Y, Li H, Zhu S, Xie Y, Wang B, He L et al (2020) Prognostic Value of right ventricular longitudinal strain in patients with COVID-19. JACC Cardiovasc Imaging. https://doi.org/10.1016/j. jemg.2020.04.014

21 Szekely Y, Lichter Y, Taieb P, Banai A, Hochstadt A, Merdler I et al (2020) The spectrum of cardiac manifestations in coronavirus disease 2019 (COVID-19)-a systematic echocardiographic study. Circulation. 142:342-353
22 Mahmoud-Elsayed HM, Moody WE, Bradlow WM, Khan-Kheil AM, Senior J, Hudsmith LE et al (2020) Echocardiographic findings in patients with COVID-19 pneumonia. Can J Cardiol 36 (8):1203-1207

23. Furian T, Aguiar C, Prado K, Ribeiro RVP, Becker L, Martinelli $\mathrm{N}$ et al (2012) Ventricular dysfunction and dilation in severe sepsis and septic shock: relation to endothelial function and mortality. J Crit Care 27 (3):319. e9-. e15

24. Parker MM, McCarthy KE, Ognibene FP, Parrillo JE (1990) Right ventricular dysfunction and dilatation, similar to left ventricular changes, characterize the cardiac depression of septic shock in humans. Chest 97 (1):126-131

25 D’Andrea A, Martone F, Liccardo B, Mazza M, Annunziata A, Di Palma E et al (2016) Acute and chronic effects of noninvasive ventilation on left and right myocardial function in patients with obstructive sleep apnea syndrome: a speckle tracking echocardiographic study. Echocardiography 33 (8):1144-1155

26 Kirkpatrick JN, Grimm R, Johri AM, Kimura BJ, Kort S, Labovitz AJ et al (2020) Recommendations for echocardiography laboratories participating in cardiac point of care cardiac ultrasound (POCUS) and critical care echocardiography training: report from the american society of echocardiography. J Am Soc Echocardiogr 33 (4):409-422e4

Publisher's Note Springer Nature remains neutral with regard to jurisdictional claims in published maps and institutional affiliations. 